\title{
Quantitative analysis of the measuring capabilities of the KT-1000 knee ligament arthrometer
}

\author{
DAVID L. KOWALK, MD, EDWARD M. WOJTYS,* MD, JEFF DISHER, MS, AND \\ PETER LOUBERT, PhD
}

\author{
From MedSport, University of Michigan Medical Center, Ann Arbor, Michigan
}

\begin{abstract}
The KT-1000 arthrometer was evaluated in vitro and in vivo to determine accuracy and quantify effects of potential error sources in clinical application. The KT1000 arthrometer in vitro accuracy was evaluated by making 30 measurements of 13 known displacements (range, +15 to $-15 \mathrm{~mm}$ ). The effect of applied force on malalignment measurements was evaluated in vitro by making repeated measurements with force applied $5^{\circ}$, $10^{\circ}$, and $15^{\circ}$ from the vertical position. The effect of malpositioning the device along the joint line was evaluated in vivo by making repeated measurements $1 \mathrm{~cm}$ proximal and $1 \mathrm{~cm}$ distal to the joint line. The KT-1000 arthrometer was accurate in vitro (average error, 0.13 $\mathrm{mm}$; SD, $0.12 \mathrm{~mm}$ ). The range of measurements increased when the angle of force application was increased. Positioning the device $1 \mathrm{~cm}$ proximal to the joint line produced larger anterior translation measurements in vivo than those at the joint line (5.8 versus 5.4 $\mathrm{mm}$ ), while positioning it $1 \mathrm{~cm}$ distal produced smaller measurements $(4.4 \mathrm{~mm})$. The KT-1000 arthrometer's accuracy indicates great potential for clinical application, but one must ensure that the displacing force is directed properly and the device is positioned accurately over the joint line.
\end{abstract}

Quantitative systems for the measurement of knee joint laxity have become important additions to the clinical and research practice of orthopaedics. ${ }^{2,4,6,7,10-13,17}$ These devices can add objective data to the physical examination of the knee. Therefore, the accuracy, reliability, and potential

\footnotetext{
"Address correspondence and reprint requests to: Edward M. Wojtys, MD, MedSport, Orthopaedic Surgery, University of Michigan Medical Center, 24 Frank Lloyd Wright Drive, POB 363, Ann Arbor, Ml 48106-0363.
}

sources of measurement error associated with the use of these devices need to be detailed. The KT-1000 knee arthrometer (MEDmetric Corp., San Diego, CA) generally is used to measure anterior and posterior tibial translation. Unfortunately, we know of no study that has evaluated the absolute accuracy and reliability of this device using known displacements; only comparisons between the KT-1000 and other commonly used arthrometers have been reported. ${ }^{111,14}$

This study assessed in vitro and in vivo the accuracy and reliability of the KT-1000 arthrometer data. Precisionmachined aluminum plates were used to produce known differentials for in vitro measurement. Repeated measurements were made to evaluate the device's accuracy and consistency over a range of known differentials. Potential sources of clinical error, such as improper force application and malalignment of the device along the joint line, were also quantified.

\section{MATERIALS AND METHODS}

Detailed descriptions of the KT-1000 arthrometer and its use were presented by Malcom et $\mathrm{al}^{12}$ and by Daniel and Stone. ${ }^{3}$ In short, the KT-1000 arthrometer is strapped to the anterior surface of the tibia so that the joint line indicator on the device is positioned over the joint line. Two reference pads, one on the tibial tubercle and the other on the patella, are displaced from a "zero" position when the tibia is translated in either an anterior or posterior direction.

The in vitro testing used known displacements for measurement that were produced by different combinations of nine aluminum plates. The plates were premeasured with a micrometer to an accuracy of $0.01 \mathrm{~mm}$. The differentials produced by various plate combinations for measurement are shown in Table 1.

During in vitro testing, the $\mathrm{KT}-1000$ arthrometer was secured to a horizontal platform with its VELCRO (VELCRO USA Inc., Manchester, NH) straps to prevent move- 
TABLE 1

$\mathrm{KT}-1000$ arthrometer in vitro measurement errors

\begin{tabular}{cccc}
\hline $\begin{array}{c}\text { Plate } \\
\text { position }\end{array}$ & $\begin{array}{c}\text { Differential } \\
(\mathrm{mm})\end{array}$ & $\begin{array}{c}\text { Mean error } \\
(\mathrm{mm})\end{array}$ & $\begin{array}{c}\text { Maximum } \\
\text { error } \\
(\mathrm{mm})\end{array}$ \\
\hline P15 & 15.011 & 0.1 & 0.6 \\
P13 & 13.106 & 0.2 & 0.6 \\
P11 & 10.998 & 0.2 & 0.5 \\
P9 & 9.068 & 0.3 & 0.5 \\
P6 & 6.020 & 0.3 & 0.5 \\
P3 & 3.048 & 0.3 & 0.6 \\
P0 & 0.025 & 0.0 & 0.4 \\
N2 & -1.880 & 0.1 & 0.4 \\
N4 & -3.988 & 0.1 & 0.3 \\
N6 & -5.918 & 0.0 & 0.3 \\
N9 & -8.966 & 0.0 & 0.2 \\
N12 & -11.938 & 0.0 & 0.3 \\
N15 & -14.961 & 0.0 & 0.3 \\
\hline
\end{tabular}

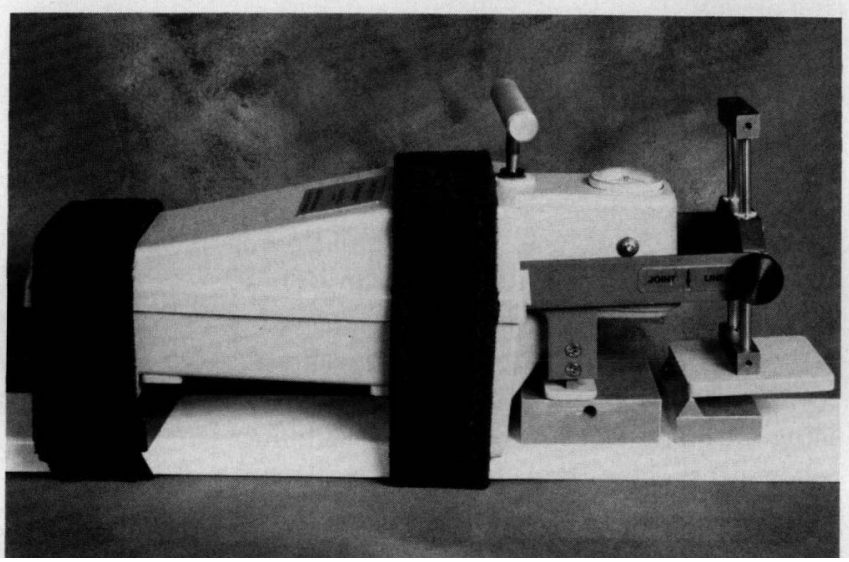

Figure 1. The in vitro KT-1000 arthrometer testing setup.

ment of the device. The reference plate was placed under the tibial tubercle pad, and the variable stack of plates was placed under the proximal edge of the patellar pad (Fig. 1). Care was taken to assure that the variable stack contacted the patellar pad at the joint line indicated by the KT- 1000 arthrometer. This ensured that the displacements being measured occurred along the intended joint line. The distal tibial contact surface of the KT-1000 arthrometer was elevated so that the proximal tibial pad could rest flat on the reference plate, allowing maximal range of measurement during testing (Fig. 1).

The differential between the tibial tubercle reference pad and the patellar reference pad was set to zero by placing equal stacks of plates (plate position at zero) under the reference pads. The KT-1000 arthrometer was lightly tapped until the indicator settled. The dial was then adjusted to read $0.0 \mathrm{~mm}$.

From the zero reference position, plates were either added or removed to produce the desired displacements. The zero setting was rechecked between each set of 6 positive and 6 negative displacements by inserting the original combination of plates used for the zero position; no adjustments were required. A series of differential measurements ranging from -15 to $+15 \mathrm{~mm}$ were repeated 30 times. Force was not applied through the handle during these measurements. For this reason, a posterior "push" was not needed between measurements to rezero the device. Means and standard deviations for all differentials measured were calculated for each displacement measured.

In vitro testing also was done to quantify the amount of error introduced into the KT-1000 arthrometer's measurements when the tibial displacing force was directed either to the right or left of the vertical position. The setup described earlier was used with the plate combination at zero and with the patellar pad held firmly in position. A goniometer was attached to the handle of the arthrometer to measure the angle at which the misguided force was applied. The displacement force was applied through a string that was tied to the center of the handle (Fig. 2). The force was added slowly at the predetermined angle, and a displacement reading was made when the 15 -pound "beep" was heard.

After each of the measurements, an attempt was made to bring the dial indicator back to a neutral position by pulling it vertically, until the 15 -pound beep was heard, and then pushing vertically in the opposite direction until another 15 pound beep was heard, as suggested by the manufacturers. The dial, however, did not always return to the starting position between measurements. For this reason, ranges of measurement observed between equal but opposite angles of pull on the KT- 1000 arthrometer handle were compared. Thirty readings were recorded at each angle of pull. Positive angles represent angles directed toward the operator's left side. The ranges between mean measurements at pull angles of $+15^{\circ}$ and $-15^{\circ},+10^{\circ}$ and $-10^{\circ},+5^{\circ}$ and $-5^{\circ}, 0^{\circ}$ from the vertical position were calculated.

In vitro testing also was performed to assess the effect of inadvertent positioning of the arthrometer, either proximally or distally to the joint line. The true joint line and 1$\mathrm{cm}$ proximal and $1-\mathrm{cm}$ distal positions were identified, and these were marked on the skin. Two subjects, 1 with an

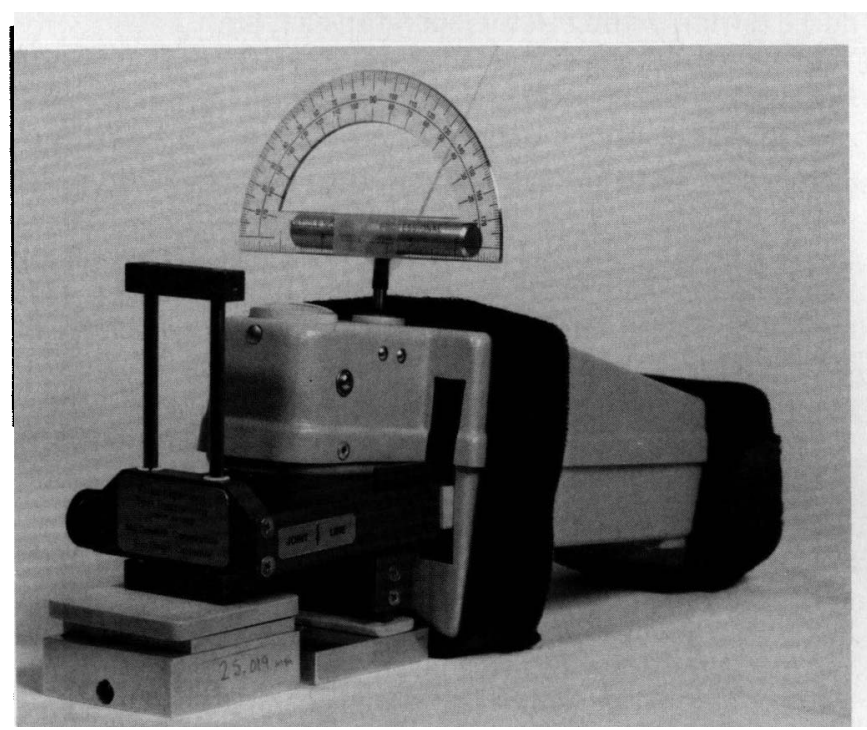

Figure 2. The KT-1000 arthrometer setup for measurement of the force application angle. 
ACL-deficient knee, were tested. For each of the 4 knees tested, 10 anterior displacement measurements were made with the KT-1000 arthrometer in the 3 positions: at the joint line, $1 \mathrm{~cm}$ proximal, and $1 \mathrm{~cm}$ distal to the joint line. After each measurement, the device was removed from the leg and then carefully was reapplied to ensure that the joint line indicator on the device was aligned properly over the desired skin marking. A paired Student's $t$-test was used to determine the statistical significance of the differences in measurements obtained in the 3 different positions.

\section{RESULTS}

Errors in the in vitro KT-1000 arthrometer measurements were calculated by subtracting the known differentials from the values obtained with the arthrometer. The mean and maximum errors in measurements for each of the known displacements are shown in Table 1. The KT-1000 arthrometer's posterior measurements were more accurate than the anterior displacements. The KT-1000 arthrometer demonstrated an overall mean error of $0.13 \mathrm{~mm}$, and an overall mean standard deviation of $0.12 \mathrm{~mm}$, with the greatest individual error being only $0.6 \mathrm{~mm}$. These measurements were significantly different from those of the micrometer $(P=0.05)$.

In vitro tests involving improperly directed loads applied to the handle of the arthrometer showed that the range of measurements between angles of pull increased as the direction of pull deviated from the vertical position (Table 2). The standard deviation of measurements at each angle of pull remained relatively constant for all angles tested (standard deviations, 0.064 to $0.092 \mathrm{~mm}$ ). It was noted that the needle deviated more from zero with positive angles of pull than with the negative angles of pull; more error was introduced during pulling to the operator's left side.

In vivo tests involving positioning of the KT-1000 arthrometer either proximal or distal to the joint line demonstrated that measurements made in a proximal position gave larger displacement values than those made with the device positioned on the joint line (overall mean, 5.77 versus 5.42 $\mathrm{mm}$ ). Measurements taken with the device distal to the joint line gave smaller displacement values (overall mean, 4.73 $\mathrm{mm}$ ). The measurements made at $1 \mathrm{~cm}$ above and below the joint line were significantly different $(P=0.05)$ from those made at the joint line in all eight comparisons.

TABLE 2

Ranges of displacement measurements between opposite angles of force application

\begin{tabular}{lcc}
\hline $\begin{array}{c}\text { Observed } \\
\text { angles }\end{array}$ & $\begin{array}{c}\text { Range } \\
(\mathrm{mm})\end{array}$ & $\begin{array}{c}\text { Range of } \\
\text { means } \\
(\mathrm{mm})\end{array}$ \\
\hline $0^{\circ}$ & $\mathbf{0 . 2}$ & 0.0 \\
$+5^{\circ}$ to $-5^{\circ}$ & $\mathbf{1 . 0}$ & 0.8 \\
$+10^{\circ}$ to $-10^{\circ}$ & $\mathbf{2 . 0}$ & 1.7 \\
$+15^{\circ}$ to $-15^{\circ}$ & $\mathbf{3 . 1}$ & 2.8 \\
\hline
\end{tabular}

\section{DISCUSSION}

Several systems have been developed to measure knee laxity objectively in both the clinical and the research settings. ${ }^{2,4,6,7,10,12,13,17}$ The ability to quantify knee laxity is important to the clinician and the researcher, but data regarding the accuracy and reliability of these systems must be available to put measurements from these devices into perspective. ${ }^{15}$ Problems encountered with the KT-1000 arthrometer in our clinical practice led us to question its accuracy and to inquire about potential sources of error for in vivo measurements. We quantified the displacement measurement capabilities of the KT-1000 arthrometer by using micrometer-measured aluminum plates as controls. By quantifying the measuring capabilities of this instrument, we hope to provide perspective for clinicians using this device.

The MEDmetric Corporation cites data from Malcom et al. $^{11,12}$ regarding the accuracy and reproducibility of its KT1000 arthrometer. Malcom et al. report that the KT-1000 arthrometer has a mean error of $0.39 \pm 0.25 \mathrm{~mm}$ when compared with measurements made by displacement transducers, and $0.16 \pm 0.44 \mathrm{~mm}$ (correlation coefficient, 0.979) when compared with a system using Steinmann pins. The KT-1000 arthrometer has been tested for reproducibility with other available knee laxity measuring devices and has been shown to perform well in comparison. Steiner et al., ${ }^{16}$ using an $89 \mathrm{~N}$ force application, calculated the $95 \%$ confidence intervals for knee laxity measurements on normal and ACL-disrupted knees: 2.3 and $2.8 \mathrm{~mm}$ for the KT-1000 arthrometer; 2.3 and $3.2 \mathrm{~mm}$ for the Stryker knee laxity tester (Stryker Corp., Kalamazoo, MI); 3.1 and $4.4 \mathrm{~mm}$ for the Acufex knee signature system (Acufex Microsurgical Inc., Norwood, MA); and 4.6 and $7.7 \mathrm{~mm}$ for the Genucom knee analysis system (FARO Medical Technologies Inc., Montreal, Canada). Although these comparisons of the KT1000 arthrometer with other measuring systems are useful, to our knowledge no data have been published before this study on the accuracy and reliability of this device in measuring known differentials over a $30-\mathrm{mm}$ range.

A separate study by Malcom et al. ${ }^{12}$ reports an intertester, side-to-side, test-retest difference of $<2 \mathrm{~mm}$ in $93 \%$ of the cases, indicating that the KT-1000 arthrometer is relatively consistent when used clinically. Hanten and Pace, ${ }^{8}$ who also reported that the $\mathrm{KT}-1000$ arthrometer is consistent in the clinical setting, demonstrated an analysis of variance interclass correlation of 0.92 for multiple testers and 0.84 for a single examiner. Wroble et al. ${ }^{19}$ reported a $90 \%$ confidence interval for repeated measurements on different days of 1.4 and $1.5 \mathrm{~mm}$, respectively, for the left and right knees with an $89 \mathrm{~N}$ force application. They found no statistically significant differences in measurements made on the same day, but they did find small, statistically significant differences in measurements made on different days. Torzilli et al. ${ }^{18}$ examined the reproducibility of the KT-1000 arthrometer by making repeated measurements on 20 subjects on different days. They found no significant difference in the meas- 
urements made on different days, and they demonstrated an average intrasubject standard deviation of 0.66 and $0.68 \mathrm{~mm}$ for anterior drawer tests performed at $30^{\circ}$ of knee flexion with 67 and $89 \mathrm{~N}$ force applications. A study by Forster et al., ${ }^{5}$ however, demonstrated a greater amount of clinical variability. They reported an intertester, single knee, testretest examination variability of $>2 \mathrm{~mm}$ in $19 \%$ of the cases. These discrepancies in clinical data warrant the investigation and quantification of potential sources of error in measurements made with the KT-1000 arthrometer.

During our testing, care was taken to position and operate the device in a manner that simulated its clinical use. Because precision-machined plates rather than actual tibial translations were measured in the in vitro testing, variations in measurements caused by changes in knee position and muscle relaxation were eliminated, thus minimizing potential sources of error.

Factors that should be considered in clinical applications, but were not factors in the first part of this study, include soft tissue compliance, knee positioning, and muscle activity. These factors may introduce variations in measurements not present in our study. The problem of soft tissue compliance may be minimal, in part because of the subcutaneous position of both the patella and tibia, which are used as contact points with the KT-1000 arthrometer. Muscle activity, however, is difficult to prevent or to quantify. Active quadriceps muscles can decrease posterior translation of the tibia just as active hamstring muscles can decrease anterior translation.

In our in vitro testing where soft tissue compliance and muscle activity were not factors, the KT-1000 arthrometer showed relatively small errors in measurement and was also very consistent (mean error, $0.13 \pm 0.12 \mathrm{~mm}$ ). The accuracy and consistency of measurements made with the KT-1000 arthrometer indicate that it has great potential for clinical use if the errors in positioning, force application, and soft tissue variables can be minimized. Significant variations in measurement can occur when the anterior-directed force is applied off-center and when the device is not aligned with the joint line.

It is important to note that while an arthrometer may be considered accurate and may have been shown to have reproducible results, values obtained for knee laxity by one type of device cannot be generalized to values obtained by another type. ${ }^{9}$

Accuracy evaluations of knee arthrometers are important for the interpretation of clinical and research data. Our investigation showed that the KT-1000 arthrometer could measure a clinically useful range of displacements $(+15$ to $-15 \mathrm{~mm}$ ) accurately and reproducibly in the in vitro setting. The data show that the range of measurements increased as the angle of force application deviated from the vertical position, and when the arthrometer was not aligned properly with the knee joint line. This result could have been anticipated. Because the KT-1000 arthrometer measures displace- ment of the tibial tubercle relative to the patella rather than making direct measurements from the tibia at the joint line, the differences in distances of these points from the axis of rotation at the heel must be accommodated by the device. When the device is positioned distal to the joint line, and the tibial tubercle pad is closer to the axis of rotation, the measured displacement is smaller than the actual displacement occurring at the joint line. The converse is true when the device is positioned proximal to the joint line. It is recommended, therefore, to avoid inconsistent or inaccurate measurements when using the KT-1000 arthrometer, one should ensure that the anteriorly directed force is in line with the vertical bar on the arthrometer handle and that the device is positioned properly over the joint line.

\section{REFERENCES}

1. Anderson AF, Lipscomb AB: Preoperative instrumented testing of anterior and posterior knee laxity. Am J Sports Med 17: 387-392, 1989

2. Blick SS, Daniel DM, Davis J, et al: Kinematics of the quadriceps stabilized anterior cruciate disrupted knee. Trans Orthop Res Soc 14: 25, 1989

3. Daniel DM, Stone ML: KT-1000 anterior-posterior displacement measurements, in Daniel DM, Akeson WH, O'Connor JJ, et al (eds): Knee Ligaments: Structure, Function, Injury, and Repair. New York, Raven Press, 1990, pp 427-447

4. Fleming B, Beynnon B, Erickson A, et al: An in vivo comparison of anterior joint laxity and strain in the anteromedial band of the anterior cruciate ligament. Trans Orthop Res Soc 14: 326, 1989

5. Forster IW, Warren-Smith CD, Tew M: Is the KT-1000 knee ligament arthrometer reliable? J Bone Joint Surg 71B: 843-847, 1989

6. Fukubayashi T, Torzilli PA, Sherman MF, et al: An in vitro biomechanica evaluation of anterior-posterior motion of the knee. J Bone Joint Surg 64A: 258-264, 1982

7. Granberry WM, Noble PC, Woods GW, et al: The accuracy and reliability of instrumented measurement of knee laxity using the Genucom knee analysis system. Trans Orthop Res Soc 14: 211, 1989

8. Hanten WP, Pace MB: Reliability of measuring anterior laxity of the knee using a knee ligament arthrometer. Phys Ther 67: 357-359, 1987

9. Highgenboten CL, Jackson A, Meske NB: Genucom, KT-1000, and Stryker knee laxity measuring device comparisons. Device reproducibility and interdevice comparison in asymptomatic subjects. Am J Sports Med 17: 743-746, 1989

10. King JB, Kumar SJ: The Stryker knee arthrometer in clinical practice. Am J Sports Med 17: 649-650, 1989

11. Malcom LL, Daniel DM, Stone ML: Instrumented knee stability measurement technique knee ligament arthrometer, model KT-1000. San Diego, CA, MEDmetric Corp, 1980

12. Malcom LL, Daniel DM, Stone ML, et al: The measurement of anterior knee laxity after ACL reconstructive surgery. Clin Orthop 196: 35-41, 1985

13. Markolf KL, Kochan A, Amstutz HC: Measurement of knee stiffness and laxity in patients with documented absence of the anterior cruciate ligament. J Bone Joint Surg 66A: 242-253, 1984

14. Sherman $\mathrm{OH}$, Markolf KL, Ferkel RD: Measurements of anterior laxity in normal and anterior cruciate absent knees with two instrumented devices. Clin Orthop 215: 156-161, 1987

15. Steiner ME, Brown C, Zarins B: Knee laxity testing: Comparison of instrumented devices and the clinical examination. Orthop Trans 13: 502, 1989

16. Steiner ME, Brown C, Zarins B, et al: Measurement of anterior-posterior displacement of the knee. A comparison of the results with instrumented devices and with clinical examination. J Bone Joint Surg 72A: 1307-1315, 1990

17. Torzilli PA, Greenberg RL, Hood RW, et al: Measurement of anteriorposterior motion of the knee in injured patients using a biomechanical stress machine. J Bone Joint Surg 66A: 1438-1442, 1984

18. Torzilli PA, Panariello RA, Forbes A, et al: Measurement reproducibility of two commercial knee test devices. J Orthop Res 9: 730-737, 1991

19. Wroble RR, van Ginkel LA, Grood ES, et al: Repeatability of the KT-1000 arthrometer in a normal population. Am J Sports Med 18: 396-399, 1990 